\title{
NEOPLASIAS MALIGNAS PRIMARIAS DEL APÉNDICE CECAL: ESTUDIO COMPARATIVO ENTRE 2 HOSPITALES REGIONALES*
}

\author{
Dr. Marcelo Zamorano D. ${ }^{1}$, Int. Manuel Quiroz F. ${ }^{2}$, \\ Al. Nicolás Drolett SF. ${ }^{3}$, Dr. Cristián Carrasco L. ${ }^{4}$
}

1 Servicio de Cirugía, Hospital Regional de Antofagasta, Universidad de Antofagasta.

2 Interno de Medicina, Facultad de Medicina y Odontología, Universidad de Antofagasta. Antofagasta.

${ }^{3}$ Estudiante de Medicina, Facultad de Medicina y Odontología, Universidad de Antofagasta. Antofagasta.

${ }^{4}$ Servicio de Anatomía Patológica, Hospital Regional de Valdivia. Universidad Austral de Chile, Valdivia. Chile.

\begin{abstract}
\section{Primary malignant tumors of appendix}

Background: Primary malignant tumors of appendix are uncommon and usually found during the pathological examination of surgical pieces of appendectomies. Aim: To report the clinical and pathological features of appendiceal malignant tumors. Material and Methods: Review of medical and pathological reports of patients subjected to an appendectomy between 1998 and 2006 in two regional hospitals. Results: Fifteen appendiceal malignant tumors in 2,687 pathological studies were detected ( $0.55 \%$ of all studies) in one hospital. In the other 10 tumors were detected in 4,939 studies $(0.2 \%)$. Nine bearers of tumors were male. In each hospital 93 and $80 \%$ of patients had an acute appendicitis, respectively. The pathology report informed a neuroendocrine tumor in 87 and $70 \%$ of patients of each hospital, respectively, followed by non-Hodgkin lymphoma and adenocarcinoma. In nine patients a staging study was performed. Four patients died during follow up, one of them due to tumor disseminations. Conclusions: Although appendiceal tumors appear in only $0.3 \%$ of all appendectomies, the pathological study of the excised appendix is fundamental for the diagnosis.

Key words: Appendix, appendectomy, tumor.
\end{abstract}

\section{Resumen}

Introducción: Las neoplasias malignas primarias del apéndice cecal son infrecuentes, generando un gran problema diagnóstico, evidenciándose la mayoría de las veces de forma incidental en el estudio histopatológico (EH). Nuestro objetivo es analizar y comparar las características epidemiológicas, clínicas, quirúrgicas e histológicas de las neoplasias malignas primarias apendiculares en dos hospitales regionales de alta prevalencia en cáncer. Materiales y Métodos: Se realizó un estudio retrospectivo de serie de casos,

*Recibido el 13 de enero de 2014 y aceptado para publicación el 21 de abril de 2014.

Los autores no refieren conflictos de interés.

Correspondencia: Dr. Marcelo Zamorano D. zamoranomarcelo@gmail.com 
se seleccionó pacientes con diagnóstico de neoplasia apendicular maligna en los Hospitales Regionales de Antofagasta (HRA) y Valdivia (HRV) entre 1998-2006, se excluyeron pacientes con neoplasias malignas secundarias. Los datos se analizaron mediante estadística descriptiva y $\chi^{2}$. Resultados: En HRA se pesquisaron $15(0,55 \%)$ casos de tumores malignos primarios en $2.687 \mathrm{EH}$, en HRV se encontraron $10(0,20 \%)$ casos en $4.939 \mathrm{EH}$. Siendo el universo muestral de 25 pacientes. En HRA, 8 fueron en hombres y 7 en mujeres, con edad media de 30 años. En HRV fueron 1 y 9 respectivamente, con edad media de 37,4 años. El 93\% y el $80 \%$ se presentaron como cuadro apendicular agudo respectivamente. La histopatología evidenció Tumor Neuroendocrino (NET) en $87 \%$ y $70 \%$ respectivamente, seguido por Linfoma no Hodgkin y adenocarcinoma. Se realizó estudio de extensión en 4 y 5 pacientes respectivamente, cuatro pacientes fallecieron en el período de seguimiento, uno a causa de diseminación. Discusión: En ambos centros la presentación clínica fue similar, el NET fue el más frecuente. La incidencia fue significativamente mayor en HRA, p $<0,05$. El estudio histopatológico rutinario es fundamental para el diagnóstico y tratamiento.

Palabras clave: Neoplasias del sistema digestivo, neoplasias apendiculares, abdomen agudo.

\section{Introducción}

$\mathrm{Al}$ analizar las estimaciones de mortalidad por cáncer en Chile, 8 regiones superan la tasa media nacional, ubicándose en primer y segundo lugar, Antofagasta y La Región de los Ríos respectivamente ${ }^{1}$. Por un lado, las mineras en zonas desérticas del norte están acompañadas de altos índices de radiación ultravioleta (UV) e importantes depósitos de arsénico, y por otro lado, la actividad agrícola y ganadera del sur, expuestos a trabajos al aire libre, con exposición a rayos UV y sustancias químicas como plaguicidas, proponen a estas regiones como ciudades apropiadas para estudiar el cáncer ${ }^{2}$.

Las neoplasias malignas del apéndice cecal corresponden a una patología extremadamente infrecuente, representando un $0,2 \%$ a $0,5 \%$ de todos los tumores de origen gastrointestinal y un $0,9 \%$ a $1,4 \%$ de las apendicetomías debido a un cuadro apendicular agudo ${ }^{3-7}$.

Esta patología se presenta generalmente como un cuadro de apendicitis aguda, siendo el diagnóstico generalmente un hallazgo producto de la cirugía, más aún, en la mayoría de los casos, se realiza con el estudio histopatológico sin siquiera sospecharlo en el intraoperatorio, lo que conlleva un diagnóstico tardío y con ello un peor pronóstico ${ }^{5,6}$.

Entre los tumores apendiculares malignos, el de mayor prevalencia es el NET, antes llamado tumor carcinoide, encontrándose hasta en el $70 \%$ de todos los casos, con una incidencia aproximada de 1,5\% y prevalencia cercana a $0,30 \% \%^{1-3,5,8,9}$.

Los NET se distribuyen en todo el sistema digestivo, aproximadamente el $19 \%$ se localizan en apéndice cecal. Otros tumores primarios como el adenocarcinoma y el cistoadenoma mucinoso son menos frecuentes ${ }^{10,11}$.

El objetivo del presente estudio fue analizar y comparar las características clínicas, quirúrgicas, histopatológicas y evolución de pacientes diagnos- ticados con neoplasias malignas primarias del apéndice cecal en el Hospital Clínico Regional de Antofagasta y Hospital Clínico Regional de Valdivia.

\section{Definiciones}

Los NET son tumores con diferenciación neuroendocrina, Landry fue quien planteó su etapificación o TNM para localización apendicular, este tipo de tumor presenta cuatro subtipos histológicos entre los que destacan los tumores de células espumosas y tumores de células enterocromafines ${ }^{12}$.

Los adenocarcinomas representan neoplasias epiteliales malignas, destacan tres tipos histológicos: adenocarcinoma tubular, adenocarcinoma mucinoso y carcinoma con células en anillo de sello, esta diferenciación no reviste diferencias clínicas significativas.

El grado de diferenciación tumoral se estableció mediante microscopia y en algunos casos con la determinación de Ki67 (principalmente en HRV). Se estableció la diferenciación con los criterios del American Joint Commission on Cancer, indicando grado $\mathrm{X}$ cuando no pudo evaluarse, hasta grado 4 o tumor indiferenciado cuando más del $75 \%$ de las células fueron inmaduras.

\section{Materiales y Métodos}

Se realizó un estudio de serie de casos, retrospectivo, comparando los resultados entre dos centros hospitalarios de Chile; Hospital Regional de Antofagasta y el Hospital Regional de Valdivia.

\section{Pacientes estudiados}

Se incluyó pacientes apendicectomizados entre 1998 y 2006, seleccionando a los pacientes con diagnóstico de neoplasia maligna apendicular primaria. Excluyéndose los pacientes con neoplasias apendiculares secundarias y tumores benignos. 
Tabla 1. Tumores malignos primarios según género y centro hospitalario

\begin{tabular}{|lccc|}
\hline & Antofagasta & Valdivia & 9 \\
Total apendicectomías & 2.687 & 4.939 & - \\
Tumores malignos primarios del apéndice cecal & $15 / 2687$ & $10 / 4939$ & $0,02(\mathrm{~S})$ \\
Sexo Masculino/Femenino & $8 / 7$ & $1 / 9$ & $0,016(\mathrm{~S})$ \\
\hline
\end{tabular}

S: Significativo.

\section{Método}

Se revisaron las historias clínicas, los protocolos operatorios y los estudios anátomo-patológicos.

Se recolectaron y tabularon los datos demográficos, cuadro clínico, conducta quirúrgica y sospecha preoperatoria e intraoperatoria, características anátomo-patológicas, evolución y causa de muerte en caso de que hubiera. Se confirmó el estado vital de los pacientes mediante el registro nacional de identificación.

\section{Cálculos estadísticos}

Se realizó una base de datos en Filemaker Pro $11 \circledR$ y la estadística descriptiva se analizó mediante el software XLSTAT $9 \circledR$ empleando $\chi^{2}$.

\section{Resultados}

En el período desde 1998 hasta el año 2006, se registraron un total de 2.687 reportes histopatológicos de apéndices extirpados en el HRA, encontrándose 15 casos de pacientes con tumores malignos primarios del apéndice cecal. En el HRV, de un total de 4.939 apendicetomías, se pesquisaron 10 pacientes con tumor maligno apendicular predominando en pacientes de sexo femenino (Tabla 1).

\section{Presentación clínica y sospecha diagnóstica}

El cuadro clínico más frecuente fue la apendicitis aguda en un $93 \%$ y $80 \%$ en el HRA y HRV, un paciente en HRA se presentó con cuadro de masa palpable y dolor abdominal, y dos pacientes en HRV se presentaron con cuadros catalogados como obstrucción intestinal.

En ningún centro se realizó diagnóstico preoperatorio y sólo se sospechó la presencia de tumor en dos pacientes durante el intraoperatorio en el HRV, a diferencia del HRA donde no hubo sospecha.

El diagnóstico definitivo en ambos centros se confirmó en el informe histopatológico en un 100\% de los casos. En el HRV se realizó inmunohistoquímica en un $80 \%$ de los casos a diferencia del HRA donde sólo se empleó en un paciente.

\section{Histopatología}

Respecto a la clasificación histopatológica, en el
Tabla 2. Comparación histopatológica entre ambos centros hospitalarios

\begin{tabular}{|c|c|c|c|}
\hline & HRA & HRV & $\mathbf{P}$ \\
\hline $\begin{array}{l}\text { Tipo histológico } \\
\text { Neuroendocrino } \\
\text { Linfoma no Hodgkin } \\
\text { Adenocarcinoma }\end{array}$ & $\begin{array}{l}87 \%(13) \\
6,60 \%(1) \\
6,60 \%(1)\end{array}$ & $\begin{array}{l}80 \%(8) \\
10 \%(1) \\
10 \%(1)\end{array}$ & $\begin{array}{l}0,68(\mathrm{NS}) \\
0,78(\mathrm{NS}) \\
0,78(\mathrm{NS})\end{array}$ \\
\hline $\begin{array}{l}\text { Infiltración } \\
\text { Submucosa } \\
\text { Muscular } \\
\text { Subserosa } \\
\text { Todo el espesor }\end{array}$ & $\begin{array}{rr}13 \% & (2) \\
60 \% & (9) \\
20 \% & (3) \\
7 \% & (1)\end{array}$ & $\begin{array}{l}10 \%(1) \\
40 \%(4) \\
20 \%(2) \\
30 \%(3)\end{array}$ & $\begin{array}{l}0,81(\mathrm{NS}) \\
0,35(\mathrm{NS}) \\
1 \quad(\mathrm{NS}) \\
0,18(\mathrm{NS})\end{array}$ \\
\hline
\end{tabular}

NS: No significativo.

Tabla 3. Localización y tamaño del tumor apendicular

\begin{tabular}{|lccc|}
\hline & HRA & HRV & p \\
Ubicación & & & \\
Tercio distal & $93 \%(13)$ & $90 \%(9)$ & $0,81(\mathrm{NS})$ \\
Tercio medio & $7 \%(2)$ & $10 \%(1)$ & $0,81(\mathrm{NS})$ \\
Tercio proximal & 0 & 0 & \\
Tamaño & & & \\
Mayor a 20 mm & 1 & 2 & $0,38(\mathrm{NS})$ \\
\hline
\end{tabular}

Mm: milímetros; HRA: Hospital Regional de Antofagasta, HRV: Hospital Regional de Valdivia, NS: No significativo.

HRA, el $87 \%$ correspondió a NET, mientras que en el HRV el NET representó el 80\% (Tabla 2).

La ubicación del tumor apendicular fue en su mayoría en la región distal del apéndice cecal (Tabla 3). La media del tamaño fue de $15 \mathrm{~mm}$ y $14 \mathrm{~mm}$ en el HRA y HRV respectivamente, encontrándose un tumor por sobre los $2 \mathrm{~cm}$ en HRA y dos en el HRV.

Se realizó estudio de diseminación y comité oncológico en 4 pacientes en el HRA y 5 en el HRV. De los pacientes con estudio de extensión en HRA, en un caso se decidió control anual con TC de abdomen y pelvis y en los otros 3 casos se decidió Hemicolectomía derecha; un caso de NET de $3,4 \mathrm{~cm}$ y dos casos de adenocarcinoma de 2,7 y $3,6 \mathrm{~cm}$ respectivamente, en ninguno de los casos la pieza operatoria demostró metástasis ni invasión 
linfo-vascular, la linfadenectomía fue negativa para metástasis linfonodales. A estos pacientes se indicó control clínico y con TC de abdomen y pelvis cada 6 meses durante los primeros dos años.

El tiempo medio de control fueron 18 meses. El seguimiento en el HRV se realizó en todos los pacientes y fue desde los 4 meses a 93 meses.

La mortalidad estuvo presente en cuatro casos, dos en Antofagasta y dos en Valdivia. En Antofagasta un caso de falla multisistémica post operatoria y en otro caso infarto agudo al miocardio a 6 meses de control ambulatorio. En Valdivia, en un caso la mortalidad fue debida a complicaciones sépticas durante el post operatorio y en un caso debido a diseminación de la enfermedad. Todos los casos de mortalidad se registraron en adultos mayores de 65 años con patologías crónicas cardiovasculares. Los pacientes operados que se mantuvieron en control no presentaron recidiva y no se registró mortalidad por causa tumoral.

\section{Discusión}

Las neoplasias primarias del apéndice cecal son extremadamente infrecuentes, lo cual dificulta su sospecha y diagnóstico precoz ${ }^{14}$. Es así como en este estudio no se realizó el diagnóstico en el preoperatorio y se sospechó solamente en dos casos de los 25 operados $(8 \%)$.

En los artículos extranjeros se presentan entre el $0,9 \%$ y $1,4 \%$ del total de las apendicectomías, mientras que en nuestro estudio se registraron prevalencias menores, no obstante, concuerdan con los registros nacionales, que señalan cifras entre $0,007 \%$ y $0,8 \% 0^{5,17-19}$.

En el HRV, el tumor apendicular fue el más frecuente en el género femenino concordando con la literatura nacional e internacional, por otro lado, el HRA mostró distribución similar en ambos sexos.
El rango de edad fue similar a lo reportado en las series nacionales, así como la incidencia de adenocarcinoma $^{5,17-19}$.

El tumor maligno más frecuente del apéndice cecal es el NET, representando el $0,2 \%$ del total de las apendicectomías, seguidos de los adenocarcinomas que incluyen los del tipo colónico, adenocarcinoides y cistoadenocarcinomas, considerados como misceláneos $^{20}$. La tendencia en nuestro estudio no varió respecto a la literatura, predominando el NET con un $87 \%$ en el HRA y con un $80 \%$ en el HRV, destacando una mayor incidencia en relación a cuatro series nacionales ${ }^{5,18,19,21}$ (Tabla 4). Se encontró un Linfoma no Hodgkin en cada centro hospitalario, siendo éste una rareza en la literatura existente ${ }^{22,23}$.

Observamos baja utilización de técnicas de inmunohistoquímica para certificar el diagnóstico en este tipo de patologías, lo que puede explicarse por la baja disponibilidad en los Hospitales durante el período de estudio.

Los tumores menos frecuentes, como los adenocarcinomas y los linfomas no Hodgkin, son de peor pronóstico en comparación con los $\mathrm{NET}^{8}$.

El diagnóstico es complejo dada su presentación clínica, dificultando el correcto diagnóstico preoperatorio e incluso intraoperatorio. En el HRV sólo se logró sospechar en el intraoperatorio en dos pacientes, a su vez, en HRA no hubo sospecha pre $\mathrm{o}$ intraoperatoria.

Dado los elementos diagnósticos de bajo rendimiento, como el cuadro clínico, exámenes de laboratorio y técnicas imagenológicas, la histopatología es la herramienta fundamental de pesquisa de este cuadro y determinación de la conducta a seguir ${ }^{24}$.

En cuanto a las características del tumor, su ubicación más habitual es en la región distal, al igual que en nuestra serie y en las series nacionales existentes ${ }^{5,18,19,21}$. Esto es importante para determinar la conducta y el tratamiento del paciente ${ }^{25}$.

Tabla 4. Comparación de series nacionales

\begin{tabular}{|c|c|c|c|c|c|c|c|}
\hline Serie & Región & $\begin{array}{c}\text { Año } \\
\text { publicación }\end{array}$ & Apendicectomías & TAMP $^{1}$ & Hombres & Mujeres & NET \\
\hline Beltrán y cols. & $\mathrm{IV}^{2}$ & 2013 & 8.976 & $0,3 \% \quad(30)$ & $30 \% \quad(9)$ & $70 \%(21)$ & $63 \% \quad(19)$ \\
\hline Venturelli y cols. & $\mathrm{X}^{3}$ & 2010 & 6.093 & $0,27 \%(17)$ & $53 \% \quad(9)$ & $47 \% \quad(8)$ & $64,7 \%(11)$ \\
\hline Butte y cols. & $\mathrm{RM}^{4}$ & 2007 & 8.125 & $0,61 \%(50)$ & $42 \%(28)$ & $58 \%(39)$ & $55,6 \%(38)$ \\
\hline Zamorano y cols. & $\mathrm{II}^{5}$ & 2014 & 2.687 & $0,55 \%(15)$ & $53 \% \quad(8)$ & $47 \% \quad(7)$ & $87 \% \quad(13)$ \\
\hline Zamorano y cols. & $\mathrm{XIV}^{6}$ & 2014 & 4.939 & $0,20 \%(10)$ & $10 \% \quad(1)$ & $90 \% \quad(9)$ & $80 \%$ \\
\hline
\end{tabular}

${ }^{1}$ TAMP: Tumores apendiculares malignos primarios. ${ }^{2}$ Hospital de la Serena, Hospital de Coquimbo y Hospital de Ovalle, período 2005 a 2011. ${ }^{3}$ Hospital Base Osorno, período 1998 a 2008. ${ }^{4}$ Hospital Clínico de la Pontificia Universidad Católica de Chile, período 1981 a 2006 (67 pacientes con tumores apendiculares). ${ }^{5}$ Hospital Clínico Regional de Antofagasta Dr. Leonardo Guzmán, período 1998 a 2006. ${ }^{6}$ Hospital Clínico Regional de Valdivia, Período 1998 a 2006. 
La conducta quirúrgica en las neoplasias del apéndice cecal son bastantes contradictorias y aún no existe acuerdo que permita orientar al cirujano sobre cuál es el tratamiento ideal ${ }^{18}$. Se recomienda realizar hemicolectomía derecha en pacientes con NET cuyo tamaño supere los $2 \mathrm{~cm}$, infiltre mesoapéndice, exista compromiso del ciego o presente alto índice mitótico, sin embargo, no se ha llegado a un consenso en los criterios ${ }^{26}$. A pesar del desacuerdo, la sobrevida de este tipo de cáncer apendicular alcanza el $99 \%$ a los 5 años.

La evidencia más fuerte existente hasta el momento se basa en la serie más importante publicada sobre $\mathrm{NET}^{9}$, que observó durante el seguimiento por más de 28 años, sólo una recurrencia regional y metástasis en un paciente con un NET mayor a $2 \mathrm{~cm}$. Por lo que recomendaron apendicectomía como tratamiento único en tumores menores a 2 $\mathrm{cm}$; en tumores mayores a $2 \mathrm{~cm}$ se recomienda la hemicolectomía derecha en pacientes jóvenes con bajo riesgo quirúrgico, lo msmo en caso de invasión vascular o compromiso del meso apendicular ${ }^{18,27,28}$.

La hemicolectomía derecha, sin embargo, ha demostrado ser beneficiosa en adenocarcinomas, aumentando la supervivencia incluso cuando los tumores miden menos de $2 \mathrm{~cm}^{29}$. Sin embargo, pese a esto, algunos estudios registran sobrevidas desalentadoras de $40 \%$ a $50 \%$ a los 5 años ${ }^{18}$.

\section{Conclusión}

La incidencia de neoplasias malignas primarias de apéndice cecal es significativamente mayor en el HRA respecto al HRV, estas diferencias podrían orientar hacia futuras investigaciones buscando posibles factores de riesgos asociados a las diferentes regiones. En relación a las series nacionales, Antofagasta destaca con una incidencia de NET cercana al $87 \%$ de los casos, mientras en series nacionales no sobrepasa el $65 \%$, lo que también indicaría diferencias importantes que podrían ser motivo de estudios posteriores. El diagnóstico de esta neoplasia reviste una especial complejidad, por lo tanto, se debe realizar un estudio histopatológico en forma rutinaria a las apéndices cecales extirpadas, ya que, en nuestro estudio, fue la única forma de realizar un correcto diagnóstico.

\section{Referencias}

1. MINSAL. Primer informe de registros poblacionales de cáncer en Chile, quinquenio 2003-2007, 2008.

2. Itriago L, Silva N, Cortés G. Cáncer en Chile y el mundo: una mirada epidemiológica, presente y futuro. Rev Med Clin Condes. 2013;24:531-52.
3. Rodríguez Z, Casaus A, Matos M. Adenocarcinoma primario del apéndice vermiforme. Rev Cubana Cir. 2010;49:58-66.

4. Alexiou K, Sikalias N, Demonakou M, Mylona S, Triantafyllis V, Kalogirou A, et al. Mucinous adenocarcinoma of the appendix presenting with atypical symptomatology and presence of pseudomyxoma peritonei: a case report. Cases J. 2009;2:9089.

5. Butte J, García Huidobro M, Torres J, Salinas M, Duarte I, Pinedo G, y cols. Tumores del apéndice cecal. Análisis anatomoclínico y evaluación de la sobrevida alejada. Rev Chil Cir. 2007;59:217-22.

6. Esmer-Sánchez DD, Martínez-Ordaz JL, Román-Zepeda P, Sánchez-Fernández P, Medina-González E. Tumores apendiculares: Revisión clínico-patológica de 5.307 apendicectomías. Cir Ciruj. 2004;72:375-8.

7. Lee WS, Choi ST, Lee JN, Kim KK, Park YH, Baek $\mathrm{JH}$. A retrospective clinicopathological analysis of appendiceal tumors from 3,744 appendectomies: A singleinstitution study. Int J Colorectal Dis. 2011;26:617-21.

8. Ruiz-Tovar J, Teruel DG, Castiñeiras VM, Dehesa AS, Quindós PL, Molina EM. Mucocele of the appendix. World J Surg. 2007;31:542-8.

9. Moertel CG, Weiland LH, Nagorney DM, Dockerty MB. Carcinoid tumor of the appendix: Treatment and prognosis. N Engl J Med. 1987;317:1699-701.

10. Hof KH, van der Wal HC, Kazemier G, Lange JF. Carcinoid tumor of the appendix: An analysis of 1485 consecutive emergency appendectomies. J Gastrointest Surg. 2008;12:1436-8.

11. Buchholtz M, Zúñiga JM, Valdés F, Fernández R, Neveu R, Trujillo C, y cols. Carcinoide gastrointestinal: Experiencia de 7 años en el Instituto Nacional del Cáncer (2000-2006). Rev Chil Cir. 2010;62:480-5.

12. Landry CS, Woodall C, Scoggins CR, McMasters KM, Martin RC. Analysis of 900 appendiceal carcinoid tumors for a proposed predictive staging system. Arch Surg. 2008;143:664-70.

13. Englehardt R, Durrani N, Mittal V. Management and Outcomes in Primary Tumors of the Appendix. Journal of Cancer Therapy 2010;1:174-80.

14. Sánchez D, Martínez J, Román P. Tumores apendiculares. Revisión clínico-patológica de 5.307 apendicectomías. Cir Ciruj. 2004;72:375-8.

15. O'Donnell M, Badger S, Beattie G. Malignant neoplasms of the appendix. Int J Colorectal Dis. 2007; 22:1239-48.

16. Tchana-Sato V, Detry O, Polus M. Carcinoid tumor of the appendix: A consecutive series from 1,237 appendectomies. World J Gastroenterol. 2006;12:6699-701.

17. Tapia O, Manterola C, Villaseca M, Araya J, Guzmán P, Roa J. Descripción clínico-morfológica y factores pronósticos en carcinomas de apéndice cecal: Estudio de cohorte. Rev Chil Cir. 2010;62:255-61

18. Venturelli M, Cáceres P, Meza A, Cárcamo I, Garcés Q. Tumores apendiculares en 6.093 Apendicecto- 
mías de urgencias. Sobrevida a 5 años. Rev Chil Cir. 2010;62:594-99.

19. Gutiérrez L, Carreño L, Ávila P, Sciaraffia C, Jáuregui $\mathrm{C}$, Oñate J, y cols. Análisis de pacientes apendicectomizados con anatomía patológica negativa para apendicitis aguda. Revista Hospital Clínico Universidad de Chile 2002;13:14-7.

20. Iñaguazo D, Mora J, Carrillo L. Neoplasias del apéndice: reporte de un caso de Adenocarcinoma. Gamo 2007:6;24-6.

21. Beltrán M, Tapia R, Madariaga J, Díaz R, Larraín C, Jaramillo L, y cols. Tumores malignos del apéndice cecal en pacientes operados por apendicitis en la IV Región de Chile. Rev Chil Cir. 2013;65:509-14

22. Murguía M, Serrano M, Bolaños M y Mendoza S. Linfoma primario del apéndice cecal con presentación clínica de apendicitis aguda. Rev Esp Patol. 2012;46:1015.

23. Ruoff C, Hanna L, Zhi W, Shahzad G, Gotlieb V, Wasif SaifM. Cancer of the Appendix: Review of the Literatu- res. ISRN Oncology 2011, Article ID 728579, 6 pages.

24. Chen H, Lee Y, Chou A. Primary appendiceal malignancy: A clinicopathologic study. Kaohsiung J Med Sci. 2006;22:618-25.

25. Uherek F, Barría C, Larraín C. Carcinoide apendicular. Comunicación de 6 casos y actualización del tema. Cuad Cir. 2004; 18:52-6.

26. Fornaro R, Frascio M, Sticchi C, De Salvo L, Stabilini $\mathrm{C}$, Mandolfino F, et al. Appendectomy or right hemicolectomy in the treatment of appendiceal carcinoid tumors? Tumori 2007;93:587-90.

27. Bamboat ZM, Berger DL. Is right hemicolectomy for $2.0 \mathrm{~cm}$ appendiceal carcinoids justified? Arch Surg. 2006;141:349-52.

28. Castañeda LE, Valanci S, Belmonte C. Incidencia de tumores del apéndice en el Centro Médico ABC. An Med. 2009;54:201-5.

29. Nitecki S, Wolff B, Schlinkert R, Sarr M. The natural history of surgically treated primary adenocarcinomas of the appendix. Ann Surg. 1994;219:51-7. 